\title{
IoT based Smart City Bin
}

\author{
Roshni Bhandari \\ Asistant Professor \\ Computer Department \\ S.S.Agrawal Institute of Engieering \\ \& Technology
}

\author{
Rathod Swapnil \\ Student \\ B.E. Semester-VIII \\ Computer Department \\ S.S.Agrawal Institute of Engineering \\ \& Technology
}

\author{
Singh Nidhi \\ Student \\ B.E. Semester-VIII \\ Computer Department \\ S.S.Agrawal Institute of Engineering \\ \& Technology
}

\author{
Desai Dhruvi \\ Student \\ B.E.Semester-VIII \\ Computer Department \\ S.S.Agrawal Institute of Engineering and \\ Technology
}

\author{
Kotadiya Harsh \\ Student \\ B.E. Semester-VIII \\ Computer Department \\ S.S.Agrawal Institute of Engineering and \\ Technology
}

\begin{abstract}
[1]Today, Waste management has been a major issue around the globe. Management of waste, proper disposal and recycling the same has been an important challenge for the various governments. The most common way of managing waste is keeping the plastic bins around the streets of the cities, but a major issue in using this approach is that the bins aren't unloaded often as they get filled up, most of the bins are flooded due to heavy disposal of wastes in them. Due to which it creates an unhygienic environment spreading diseases in the surrounding area. To overcome this situation, We have proposed an IoT based Smart City Bin, which is generally a dustbin embedded with sensors. The main purpose of our "Smart City Bin" is to send alert SMS to municipal corporation persons when the bin gets filled with waste using the GSM module interfaced with a microcontroller embedded into the bin. As soon as the municipal corporation person receives the SMS alert he/she will send the garbage collector from the specific area to unload the bin. The main purpose of this approach is the develop a smart city containing "Smart City Bin" using IoT devices.
\end{abstract}

\section{General Terms}

Alerts, SMS, Location, Admin, Municipal Corporation.

\section{Keywords}

IoT, Waste Management, GSM Module, Microcontroller.

\section{INTRODUCTION}

[1]A "Smart City Bin" assures that there is proper waste management by using less energy. It is made possible by using various sensors and modules in the Bin. Every year the Government spends crores of rupees in the process of waste management and waste recycling, but still, solid waste management has been a major issue due to lack of cutting edge technologies. By optimizing the deployment of smarter technology for waste management, activities can be carried out very efficiently to reduce operational costs.

In today's smart era, the world is connected to smart devices. The field IoT has been widely revolutionary studied and implemented over different corners of the world. It is implemented in fields like Health, Education, Security, Traffic Management, Home Automation, etc. In this paper, we have proposed a "Smart City Bin" which allows the waste management authority to keep an eye on the bins located on the streets without keeping a constant eye on them. As in the proposed system, the bins are kept in the different location of the city, whenever a bin from a particular area is filled up, it will send an SMS alert to the authority that Bin No. "XYZ" is full depends upon which bin is filled up. Many surveys are been done on the same topic and many algorithms had been found regarding Smart Waste Management. Several methods are implemented for checking the condition of the bin. Though every algorithm and techniques lack some of the features so we have tried to collect several algorithms and had tried to implement all in a single "Smart City Bin".

\section{SYSTEM OVERVIEW}

In this paper the proposed system refers to a "Smart City Bin", which uses an ultrasonic sensor to detect motion in front of the dustbin to open and close the lid of the Bin automatically without any interaction with the human hand with the lid. The ultrasonic sensor transmits waves to detect any object or hand on which the waves collide. Other than that an Ultrasonic sensor is also embedded inside the bin to check the level of the bin. If the waste in the bin reaches the threshold level, the ultrasonic sensor will detect it and an SMS alert will be sent to the municipal authority using the GSM module interfaced with Arduino UNO. The level of waste in the bin is estimated upon the readings of the ultrasonic sensor. As the distance between the ultrasonic sensor and the waste keeps on decreasing as the waste in the bin keeps on increasing. The waves transmitted by the Ultrasonic sensor collide with the waste and sends the signals back to the receiver in the ultrasonic sensor which detects the distance of the waste from the sensor and when the waste reaches a particular distance an SMS alert is sent to the municipal authority using GSM module and microcontroller(Arduino UNO).

\section{PROPOSED METHODOLOGY}

With the help of IoT, we can connect various things over a network to observe things in real time. In this system, Dustbins are arranged at different places and sensors are kept such that on getting filled up with waste sensors detect the same and sends SMS alerts to the municipal corporation authority. Two ultrasonic sensors are kept, one inside the bin and other facing outside the bin. Both the ultrasonic sensors 
are interfaced with Arduino UNO microcontrollers respectively. Each ultrasonic sensor has a different function. Here the Ultrasonic sensors send data to Arduino UNO which acts as a receiver, it takes the input from the sensors and performs some operation as a form of output.

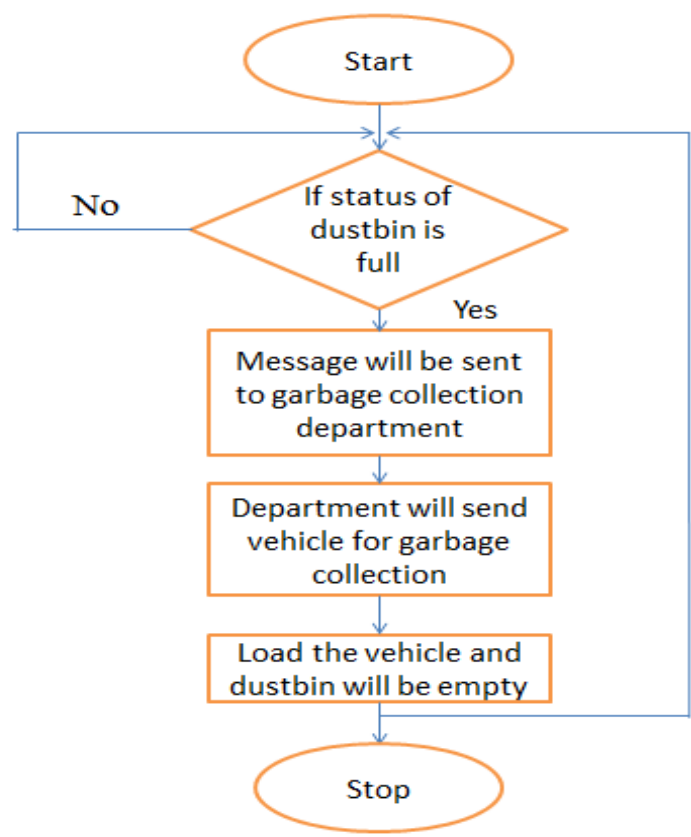

Figure 1: Flowchart for the System[2]

One of the Ultrasonic sensors is used to detect whether the dustbin is about to overflow or not, and the second ultrasonic sensor detects the hand or object in front of the bin so that it can open and close automatically.GPS tracker is used to just note down data into the database that is to be fetched on the webpage. A GPS tracker is used in the bin which is also interfaced with another Arduino UNO microcontroller, which is used from exact latitude and longitude of the bin so that further bin can be located in the google maps for exact location.

\section{IMPLEMENTATION AND SETUP}

\subsection{Main components:}

4.1.1Arduino UNO:[3]Arduino Uno is a microcontroller board based on the ATmega328P (datasheet). It has 14 digital input/output pins (of which 6 can be used as PWM outputs), 6 analog inputs, a $16 \mathrm{MHz}$ ceramic resonator (CSTCE16M0V53-R0), a USB connection, a power jack, an ICSP header, and a reset button. It contains everything needed to support the microcontroller; simply connect it to a computer with a USB cable or power it with an AC-to-DC adapter or battery to get started. Below is the figure and technical specifications for Arduino UNO.

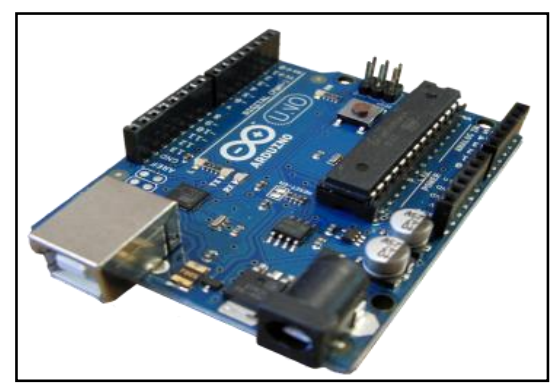

Figure 2: Arduino UNO[3]

\section{Features:}

- The operating voltage is $5 \mathrm{~V}$

- $\quad$ Digital input/output pins are 14

- $\quad$ Analog i/p pins are 6

- DC Current for each input/output pin is $40 \mathrm{~mA}$

- $\quad$ Flash Memory is $32 \mathrm{~KB}$

- CLK Speed is $16 \mathrm{MHz}$

4.1.2 Ultrasonic sesnsor(HC-SR04):[4] At its core, the HC-SR04 Ultrasonic distance sensor consists of two ultrasonic transducers. The one acts as a transmitter which converts electrical signal into $40 \mathrm{KHz}$ ultrasonic sound pulses. The receiver listens for the transmitted pulses. It works for measuring distance between $4 \mathrm{~cm}-200 \mathrm{~cm}$. If it receives them it produces an output pulse whose width can be used to determine the distance the pulse travelled. is the figure and technical specifications for Ultrasnoic sensor (HC-SR04)

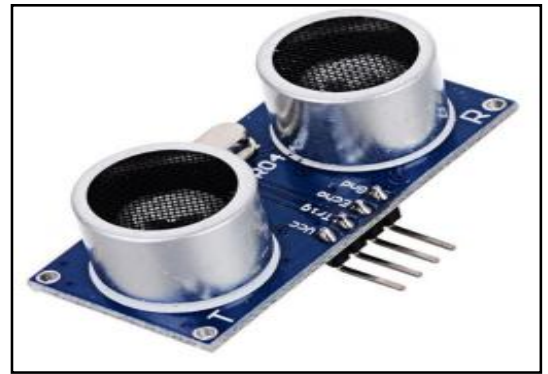

Figure 4: Ultrasonic Sensor HC-SR04[4]

Features:

- Input Voltage: $5 \mathrm{~V}$

- Current Draw: 20mA (Max)

- Digital Output: $5 \mathrm{~V}$

- Digital Output: 0V (Low)

- Working Temperature: $-15^{\circ} \mathrm{C}$ to $70^{\circ} \mathrm{C}$

- Sensing Angle: $30^{\circ}$ Cone

- $\quad$ Angle of Effect: $15^{\circ}$ Cone

- Ultrasonic Frequency: 40kHz

- Range: $2 \mathrm{~cm}-400 \mathrm{~cm}$

4.1.3 GSM SIM900A Module:[6] GSM/GPRS ModemRS232 is built with Dual-Band GSM/GPRS engineSIM900A works on frequencies 900/ $1800 \mathrm{MHz}$. The Modem is coming with RS232 interface, which allows you connect PC as well as a microcontroller with RS232 Chip(MAX232). The baud rate is configurable from 9600-115200 through AT command.. The onboard Regulated Power supply allows you to connect wide range unregulated power supply. Using this modem, you can make audio calls, SMS, Read SMS, attend the incoming calls and internet etc through simple AT commands. 


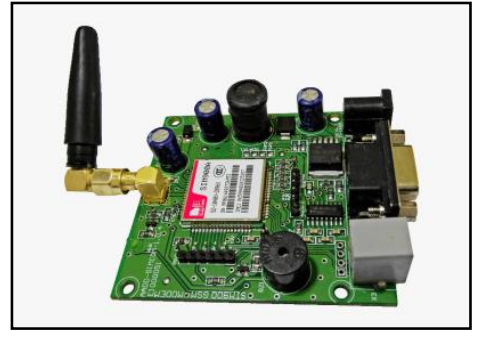

Figure 6: GSM SIM900A Module[6]

\section{Features:}

- Dual-Band GSM/GPRS 900/ $1800 \mathrm{MHz}$

- $\quad$ RS232 interface for direct communication with computer or MCU kit

- Configurable baud rate

- $\quad$ Power controlled using 29302WU IC.

- ESD Compliance.

- $\quad$ Enable with MIC and SPeaker socket.

- With slid in SIM card tray.

- With Stub antenna and SMA connector

- Input Voltage: 12V DC.

- $\quad$ High quality PCB FR4 Grade with FPT Certified.

4.1.4 GPS module SKG13:[5] The GPS Shield SKG13 is a complete GPS receiver module that features super sensitivity, ultra low power and small form factor, and a complete serial data message with position, velocity and time information is presented at the serial interface with NMEA protocol. Operating voltage: $5 \mathrm{~V}$ DC from arduino board. Compatibility with Arduino Uno, Arduino Mega 2560.

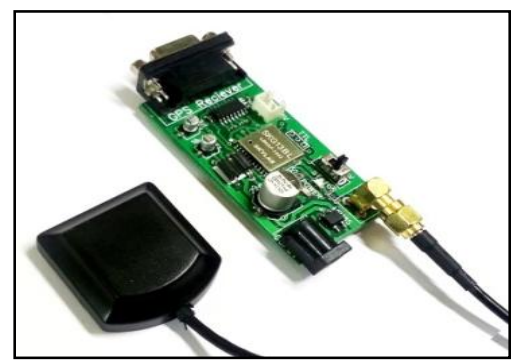

Figure 7: GPS module SKG13[5]

\section{Features:}

- Ultra high sensitivity: $-165 \mathrm{dBm}$.

- Extremely fast TTFF (Time To First Fix) at low signal level.

- Low power consumption.

- NMEA-0183 compliant protocol or custom protocol.

- Operating temperature range: 40 to 85 degree Centigrade.
4.1.5 9G Servo motor:[7]It is a motor which is used in automatic opening and closing of the lid of the "Smart City Bin".

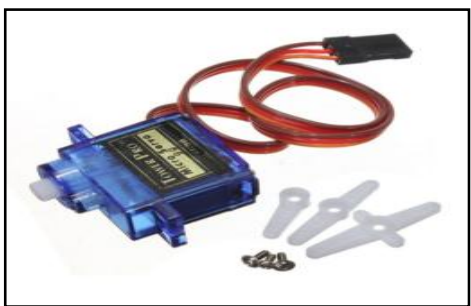

Figure 8: 9G Servo Motor[7]

Features:

- $\quad$ Micro 9g analog servo

- $\quad$ Operating Voltage: 4.8 Volts

- $\quad$ Plastic gears

- Speed: 0.12sec/60degree

Further we have also developed a webpages made with HTML, CSS and PHP, as an ADMIN LOGIN PAGE for municipal corporation authority who will receive the message that the particular bin is full. Suppose he/she gets a message that bin no.1 is full than he/she will get the coordinates of the particular bin and will search the location on the map, and will also check which person is allocated for waste management there and will tell him/her to unload the bin as soon as possible.

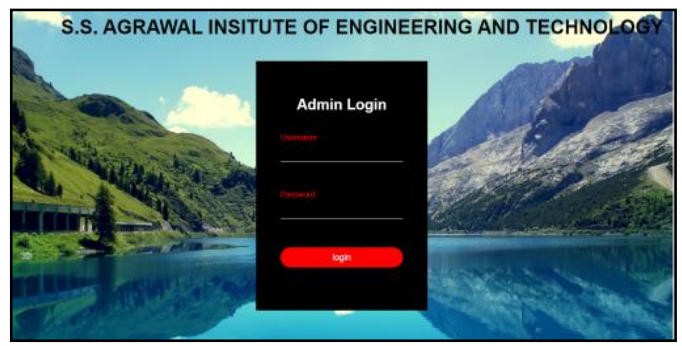

Figure 9: Admin Login Page

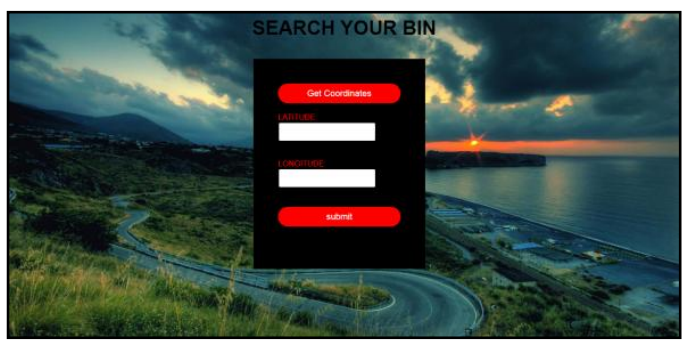

Figure 10: Searching and getting coordinates

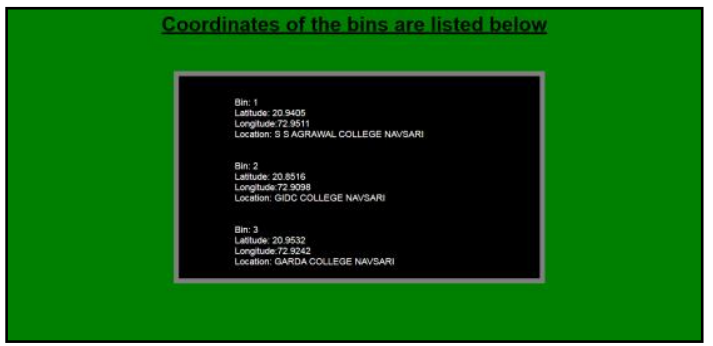

Figure 11: Coordinates Info 


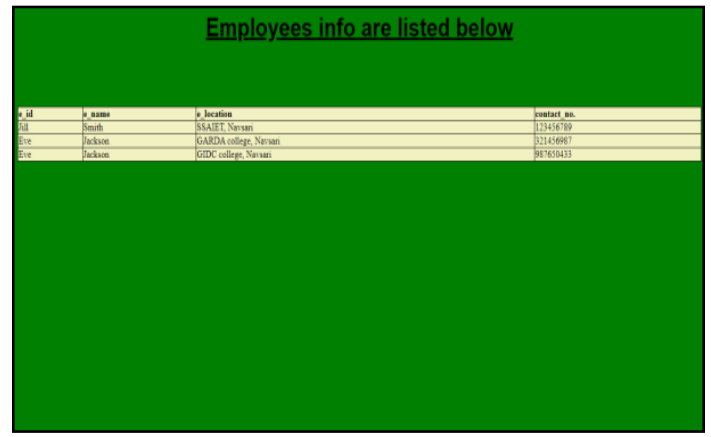

Figure 12: Employee Details

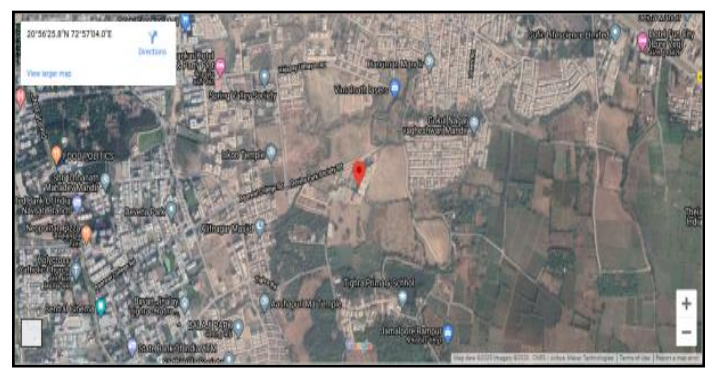

Figure 13: Google Map showing bin location

\section{RESULTS \& DISCUSSION}

We performed loading and unloading of bins many times to check whether the SMS alert is going to the municipal corporation authority or not. We loaded the bin using papers, or any other form of objects and filled the bin uptill the set range of ultrasonic sensor and as soon as the sensor detected the waste nearby it, the SMS alert went to the municipal corporation authority. Further we checked that on sensing our hand motion the lid of the bin is opening automatically or not and it seemed to be working well. We also checked that the gps receiver is also showing the correct latitude and longitude as same as the location of the dustbin. Some snapshots of the same are kept below

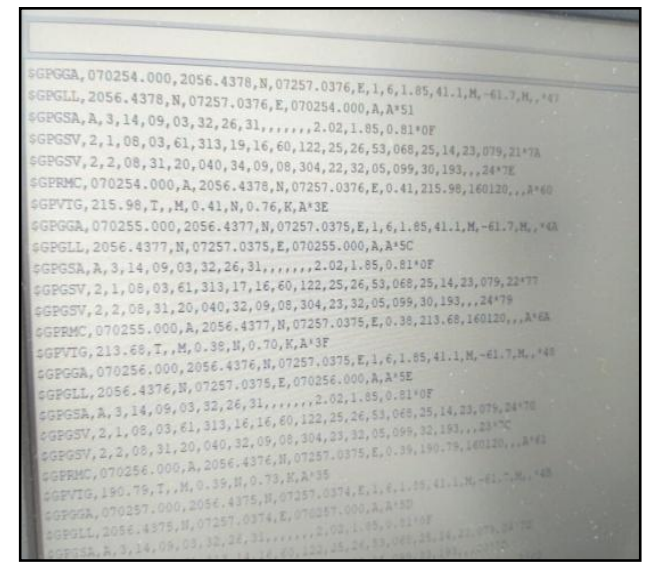

Figure 14: Getting lat long with the help of GPS receiver

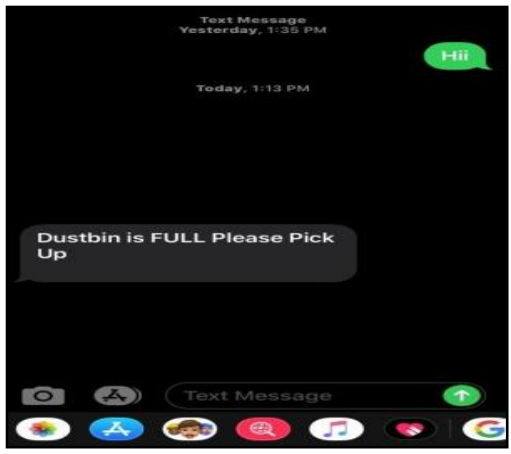

Figure 15: SMS alert on dustbin getting full

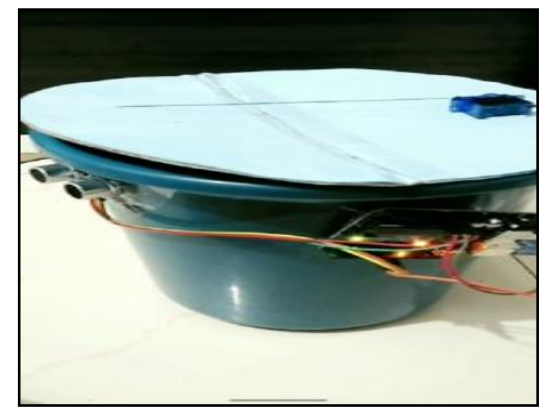

Figure 16: Dustbin Prototype

\section{CONCLUSION}

In this paper we have shown practical use of IoT in making day to day life better and hygienic by replaces normal dustbins on the streets around us by smart dustbins, Still there is lot of scope of good improvement in the same approach using different algorithms and still much more advancements can be done. For now our approach makes it more convenient for the user to use the bin as its opened and closed automatically and overflowing of the bin could be brought to an end as it sends SMS alerts on getting filled up till a threshold level. Optimizing power requirement will also be a big challenge, many tests have been performed many algorithms have been tested before proposing this approach.

\section{REFERENCES}

[1] Survey on IOT based Smart City Bin, IJCA,Roshni Bhandari, Singh Nidhi, Rathod Swapnil, Desai Dhruvi, Kotadiya Harsh, Ocotober 2019

[2] IoT based solid waste management system for smart city,Research Gate, Prashant Mulay, June 2017

[3] store.arduino.cc/usa/arduino-uno-rev3

[4] components101.com/ultrasonic-sensor-working-pinoutdatasheet

[5] graylogix.in/gpssheildskg13

[6] researchdesignlab.com/gsm-sim-900

[7] robu.in/product/towerpro-sg90-9g-mini-servo-9-gram 